\title{
ELSEVIER
}

\section{Understanding the effects of air pollution on neurogenesis and gliogenesis in the growing and adult brain}

\author{
Enrica Boda ${ }^{1,2}$, Antonello E Rigamonti ${ }^{3}$ and Valentina Bollati ${ }^{3}$
}

Exposure to air pollution - and particularly to particulate matter (PM) - is strongly associated with higher risk of neurodevelopmental disorders, poor mental health and cognitive defects. In animal models, disruption of CNS development and disturbances of adult neurogenesis contribute to PM neurotoxicity. Recent studies show that gestational PM exposure not only affects embryonic neurodevelopment, but also disturbs postnatal brain growth and maturation, by interfering with neurogenic/gliogenic events, myelination and synaptogenesis. Similarly, adult neurogenesis is affected at many levels, from neural stem cell amplification up to the maturation and integration of novel neurons in the adult brain parenchyma. The underlying mechanisms are still by and large unknown. Beyond microglia activation and neuroinflammation, recent studies propose a role for novel epigenetic mechanisms, including DNA methylation and extracellular vesicles-associated microRNAs.

\footnotetext{
Addresses

${ }^{1}$ Department of Neuroscience Rita Levi-Montalcini, University of Turin, Italy

${ }^{2}$ Neuroscience Institute Cavalieri Ottolenghi (NICO), University of Turin, Regione Gonzole, 10 - 10043 Orbassano, Turin, Italy

${ }^{3}$ Department of Clinical Sciences and Community Health, University of Milan, Milan, Italy
}

Corresponding author: Boda, Enrica (enrica.boda@unito.it)

Current Opinion in Pharmacology 2020, 50:xx-yy

This review comes from a themed issue on Neurosciences: neurogenesis

Edited by Annalisa Buffo and Stefania Ceruti

https://doi.org/10.1016/j.coph.2019.12.003

1471-4892/@ 2019 Elsevier Ltd. All rights reserved.

Exposure to air pollution is increasingly acknowledged as one of the main contributors to the global disease burden [1]. It has been estimated that in $201691 \%$ of the world population was living in places where the WHO air quality guidelines levels were not met (https:/www.who.int/news-room/ fact-sheets/detail/ambient-(outdoor)-air-quality-andhealth). Among the key air pollutants that pose health risks, particulate matter $(\mathrm{PM})$ is one of the most widespread. PM is a heterogeneous mixture of small solid or liquid particles released into the atmosphere during combustion processes or emitted by industrial activities and natural sources. PM generally comprises water soluble and insoluble components, including inorganic compounds, polycyclic aromatic hydrocarbons, heavy metals and other toxic substances, and microbial components, such as bacteria and their products of degradation (e.g. lipopolysaccharide) and viruses [2]. PM is defined according to its aerodynamic diameter, with coarse PM smaller than $10 \mu \mathrm{m}\left(\mathrm{PM}_{10}\right)$ and fine and ultrafine PM smaller than $2.5\left(\mathrm{PM}_{2.5}\right)$ or $0.1\left(\mathrm{PM}_{0.1}\right) \mu \mathrm{m}$, respectively. Thanks to their small size, when inhaled, PM particles have the capability to percolate through the respiratory tract. While $\mathrm{PM}_{10}$ is trapped in the upper airways, $\mathrm{PM}_{2.5}$ reaches the lungs and deposits in the alveolar area. Ultrafine particles could even penetrate into the blood circulation and overcome the blood-brain-barrier (BBB) $\left[3,4^{\circ}\right]$, or pass through the nasal mucosa and directly enter the brain $[5,6]$. Of note, inhaled nanoparticles have been shown to cross the placental barrier and to deposit in the fetal tissues in animal models $\left[7^{\circ}\right]$, suggesting a possible mother-to-fetus transfer of airborne ultrafine PM.

Chronic exposure to air pollution has been consistently associated with risk of cardiovascular and respiratory diseases, and different types of cancer [1]. Increasing evidence also indicates that the central nervous system (CNS) is a target for air pollution. In utero and early child exposure to high levels of air pollution, and in particular to PM, is associated with higher risk of neurodevelopmental disorders, long-lasting behavioral alterations and cognitive defects [8,9]. Moreover, during adulthood, chronic PM exposure has been associated with poor mental health, increased risk of onset and worsening of depression [9], while both short and long term exposure has been associated with cognitive/memory deterioration $\left[10^{\bullet \bullet}, 11\right]$.

Most studies in animal models that aimed at establishing a causative link between air pollution and anatomical/ functional CNS alterations, and at unveiling the underlying mechanisms, are focused on the effects of PM. In rodents, PM exposure results in neurodevelopmental, cognitive and behavioral alterations reminiscent of those observed in humans, whose extent and duration depend on PM size, doses and timing of exposure $\left[12-15,16^{\circ}, 17\right]$. Mechanistically, disruption of CNS development and of adult neurogenesis was found to contribute to PM detrimental effects, suggesting the occurrence of similar events in humans (Figure 1). 


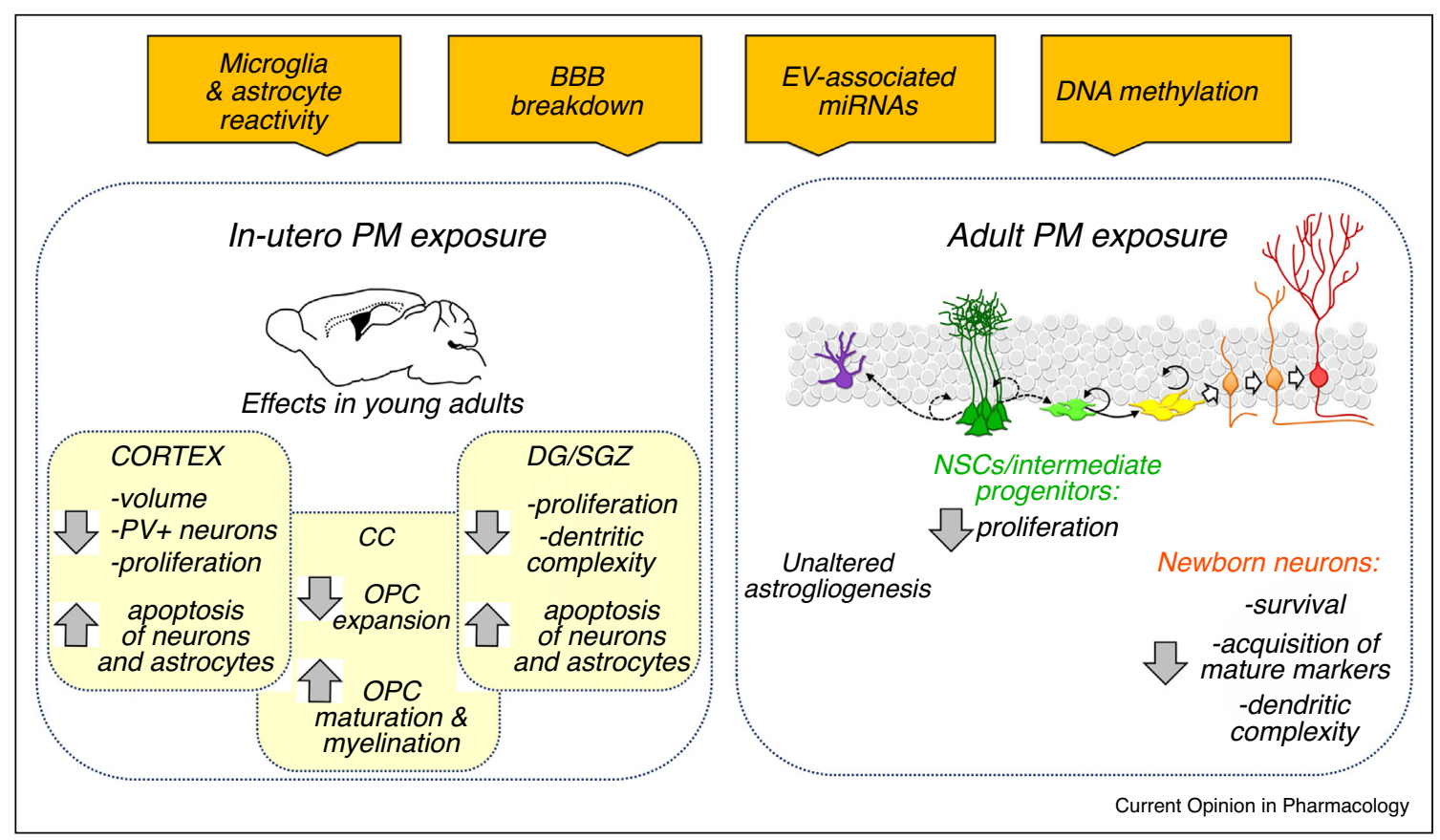

PM-induced alterations detected in the adult mouse brain following in-utero or adult exposure.

Orange boxes (above) include the proposed underlying mechanisms. BBB, blood-brain barrier; CC, corpus callosum; DG/SGZ, hippocampal dentate gyrus/subgranular zone; EV, extracellular vesicles; NSCs, neural stem cells; OPC, oligodendrocyte precursor cell; PM, particulate matter; PV, parvalbumin.

In this review, we summarize recent advancements toward the understanding of the cellular and molecular mechanisms mediating PM effects on the developmental and adult neurogenesis and gliogenesis, discuss limitations of the available studies and highlight persisting open issues.

\section{In utero and neonatal exposure to PM induces neurodevelopmental alterations in animal models}

In mice, chronic prenatal exposure to high levels of fine and ultrafine PM was reportedly associated with reduced brain weight and ventriculomegaly at birth and during the first postnatal period $[13,18]$. This is the outcome of the disruption of specific and diverse neurodevelopmental events. Exposure to diesel exhaust particles (DEP) in mouse pregnant dams throughout gestation resulted, in the offspring, in increased cortical (i.e. prefrontal cortex) and hippocampal (i.e. dentate gyrus, DG) volumes at embryonic day (E)18, which switched to decreased cortical volume and normalized hippocampal size in postnatal day $(\mathrm{P})$ 30 males (but not in females), compared to untreated animals $\left[1^{\circ}\right]$. Similarly, maternal inhalation of carbon black nanoparticles (produced by the incomplete combustion of petroleum products) resulted in an initial increase of parvalbumin-positive $(+)$ neurons in the uppermost layers of the motor cortex, followed by a large reduction at later time points [20]. These results suggest that gestational PM exposure may differentially affect distinct phases of brain development and cause an initial tissue overgrowth possibly due to neural stem cell (NSC)/progenitor overexpansion - followed by postnatal regressive events. Thus, the effects on CNS development of in utero PM exposure can be persistent and extend beyond the embryonic period. In line with this interpretation, two recent studies [12,21] have shown that chronic prenatal exposure to high dosages of $\mathrm{PM}_{2.5}$ resulted in increased neuronal and astrocyte apoptosis in the cortex and distinct hippocampal subregions, including the DG, of the offspring at P14-P30. Postnatal hippocampal neurogenesis and astrogliogenesis appeared also dramatically reduced, due to the suppression of NSC proliferation in the subgranular zone (SGZ). Similarly, parenchymal astro- and oligodendro-glia amplification was affected, as indirectly assessed by the large decrease of the proliferation marker PCNA in the cortex of P1-P30 offspring [21]. In agreement with this finding, gestational chronic exposure to fine and ultrafine particles has been associated with precocious myelination and premature oligodendroglia proliferation/differentiation switch in the corpus callosum of the adolescent offspring [13,22 $]$. Dendritic complexity [15] and number of asymmetric excitatory synapses impinging on hippocampal neurons were also significantly reduced in adolescent (P14) mice prenatally exposed to $\mathrm{PM}_{2.5}$. The remaining synapses showed altered - and 
possibly compensatory - features, including increased number of presynaptic vesicles, thickened postsynaptic density and decreased synaptic space [12].

Thus, gestational PM exposure not only affects embryonic neurodevelopment, but also disturbs postnatal brain growth and maturation, by interfering with neurogenic/ gliogenic events, myelination and synaptogenesis. Pregnancy appears to be a particularly vulnerable time window, since neonatal exposure had milder effects, and mostly affected myelination [23,24] and expression of synaptic proteins [14].

\section{PM exposure disturbs adult neurogenesis in animal models}

In the adult mouse brain, generation of new neurons continues in the subventricular zone (SVZ) of the lateral ventricles and in the SGZ of the hippocampus [25]. Adult neurogenesis in the SVZ cannot be detected in humans, whereas controversial evidence has been provided about the generation of new neurons in the adult human hippocampus $\left[26^{\bullet \bullet}, 27^{\bullet \bullet}, 28^{\bullet \bullet}\right]$. Thus, while adult hippocampal neurogenesis is implicated in cognitive processes and mood regulation in rodents [29], whether this occurs also in adult humans is highly debated. Nevertheless, adult neurogenesis in rodents recapitulates many aspects of the developmental neurogenic/gliogenic events. Therefore, the study of the mechanisms mediating PM-induced perturbations of the adult neurogenic niches is still of interest, as it can unveil critical toxicity processes operating in both developing and mature CNS.

In a recent study, acute exposure to fine DEP caused an impairment of adult neurogenesis in mice. This effect was gender-specific, with males showing fewer newlygenerated neurons in SGZ, SVZ and olfactory bulb (OB), compared to control animals, and females displaying fewer new neurons only in the $\mathrm{OB}\left[30^{\circ}\right]$. Reduced neurogenesis was a consequence of decreased proliferation of NSCs/progenitors, reduced survival of immature neurons, and altered specification/differentiation of newborn elements (i.e. reduced fraction of newborn cells expressing the mature neuronal marker NeuN 3 weeks after their generation $\left.\left[30^{\circ}\right]\right)$. Moreover, life-long exposure to concentrated water-soluble subfraction of $\mathrm{PM}_{0.2}$ dramatically reduced the number of SGZ newborn neurons -but not of newborn astrocytes- in adult male rats, which also showed contextual memory defects and depressive behaviors $\left[16^{\circ}\right]$. Thus, PM appears to negatively modulate the neurogenic events at many levels, from NSCs division up to the maturation and integration of novel neurons in the adult brain parenchyma. In line with this view, chronic inhalation of ammonium sulfate, the major inorganic component in $\mathrm{PM}_{2.5}$ (as resulting from the reaction of ammonia, mostly originating from animal farming and synthetic fertilizers, with sulfur dioxide emitted by the burning of fossil fuels [31]), diminished the dendritic complexity of immature neurons in the DG of aged rats [32]. However, in this latter study, no alteration of SGZ/SVZ NSC/progenitor proliferation and of the specification of their derivatives could be detected, highlighting a specific neurotoxicity of the distinct components of PM.

\section{Proposed mechanisms underlying the effects of PM on neurogenesis and gliogenesis}

In rodents, neuroinflammation accompanied by microglia and astrocyte activation were cardinal effects of PM exposure, whenever it occurs $\left[12-15,16^{\bullet}, 19^{\bullet}, 20,23,24,30^{\bullet}\right]$. Pharmacological treatments aimed at blocking microglia polarization - such as the peroxisome proliferator-activated receptor $\gamma(\operatorname{PPAR} \gamma)$ agonist pioglitazone - protected against PM-induced suppression of SGZ proliferation and rescued the number of newborn neurons, indicating a major role of microglia reactivity in the negative modulation of adult hippocampal neurogenesis $\left[30^{\circ}\right]$. Nevertheless, mechanistically, which activated microglia phenotype (i.e. proregenerative $\mathrm{M} 2$ versus neurotoxic $\mathrm{M} 1$ versus 'dark microglia' [33]) is favored upon/after PM exposure and how microglia activation inhibits the neurogenic events remain obscure. Beyond the release of high levels of pro-inflammatory cytokines or reactive oxygen species that can inhibit $\mathrm{NSC} /$ progenitor proliferation and alter the specification and survival of their derivatives [34], an interesting hypothesis is that PM-induced microglia activation could result in increased phagoptosis (i.e. the engulfment of immature viable neurons [35]). In line with this hypothesis, Bolton and colleagues $\left[19^{\circ}\right]$ reported increased microglia-neuron physical interactions in the cortex of the offspring of PMexposed dams.

Notably, upon prenatal and neonatal PM exposure, microglia activation and astrogliosis occurred predominantly in males $\left[19^{\bullet}, 23,24,36\right]$. Consistently, neuroinflammation was more pronounced in males than in females upon exposure to DEP during adulthood [37], in line with a more marked reduction of adult neurogenesis [30 $0^{\circ}$. This suggests that sex-dependent factors, including the hormonal background, may influence the individual's vulnerability to PM effects. Interestingly, microglia activation and neuroinflammation extended well beyond PM-exposure, when it occurred in utero, in line with a priming action of air pollution.

Moreover, what is the trigger for microglia and astrocyte activation remains elusive. Fine and ultrafine particles could enter the CNS and directly stimulate glial reactivity. Given the relatively small extension of the olfactory mucosa, it is likely that in humans - at difference with rodents - the main entrance route for PM is the blood. In line with this view, astroglia reactivity was observed predominantly around blood vessels [38]. Nevertheless, glial cells and NSCs/progenitors may be reached by a plethora of other factors - and even cells - from the 
periphery, thanks to the disruption of $\mathrm{BBB}$ integrity and increased leakage induced by $\mathrm{PM}$ exposure $\left[13,16^{\circ}\right]$. Among these elements, pulmonary cell-derived extracellular vesicles (EVs) may represent important lung-tobrain mediators of PM effects [39,40]. EVs are lipid bilayer-delimited particles, actively released from cells in response to stress. After internalization within target cells, EVs deliver their content, including proteins, lipids and miRNAs, and profoundly influence the recipient cell molecular state and function [41]. Interestingly, recent studies $[39,40]$ showed that, in humans, the miRNA cargo of plasma EVs released following PM exposure has a signature relevant for the modulation of glial cell reactivity (e.g. miR-9, involved in microglia activation and neuroinflammation [42]) and NSC/progenitor functions (e.g. miR-128, miR-302, let-7 and miR-9, regulating neural precursor proliferation and neurogenesis [43]; miR-21, miR-9, miR-200, miR-17, miR-7, miR-302c, limiting oligodendroglia differentiation or enriched in immature oligodendrocyte precursors [44]). Finally, a novel epigenetic mechanism possibly mediating PM effects on developmental and adult neurogenesis may be the regulation of DNA methylation in NSCs and their derivatives that has been shown to be responsive to extrinsic signals and to influence multiple aspects of neurogenesis from stem cell maintenance up to synaptogenesis [45]. This hypothesis is corroborated by the observation of increased DNA methyltransferase DNMT1 in the brains of male mice perinatally exposed to DEP [46 $\left.{ }^{\circ}\right]$. Notably, in human placenta, PM exposure was associated with altered methylation level of DNA repair and clock genes $\left[47^{\circ}, 48^{\circ}\right]$, which are also essential for adult and developmental neurogenesis [49-51].

\section{Concluding remarks and open issues}

Convincing evidence, obtained in animal models, shows that CNS development and adult neurogenesis are profoundly impacted by PM exposure throughout life, with significant behavioral and cognitive alterations. This field of research is still in its infancy and strenuous efforts are still needed to clarify the precise mechanisms by which PM affects neurodevelopmental events and adult neurogenesis, and the molecular substrates of gender and time window-specific differences in PM sensitivity. Available mechanistic studies have frequently exploited heterogeneous PM dosages, composition, administration modalities and timing. This scenario has so far impeded a complete understanding of the processes subserving PM effects. Nevertheless, research on the effects of PM on other systems has greatly advanced in the last years and identified interesting candidate mechanisms that could be also at the basis of PM neurotoxicity.

\section{Authors' contribution}

Enrica Boda: Conceptualization; Investigation; Visualization; Writing - original draft; Writing — review \& editing.
Antonello E. Rigamonti: Conceptualization; Investigation; Visualization; Writing - original draft; Writing review \& editing.

Valentina Bollati: Conceptualization; Investigation; Visualization; Writing — original draft; Writing — review \& editing.

\section{Conflict of interest statement}

The authors declare no conflict of interest. The funding sponsors had no role in the interpretation of data or in the writing of the manuscript.

\section{Acknowledgements}

We apologize to colleagues whose work we could not include due to space limitations. We thank Dr. Sara Bonzano for precious help in figure graphics. Our work is supported by the Individual funding for basic research (Ffabr) granted by the Italian Agency for the Evaluation of University and Research, and local funds by University of Turin to EB. This study was also supported by Ministero dell'Istruzione, dell'Università e della RicercaMIUR project 'Dipartimenti di Eccellenza 2018-2022' to Dept. of Neuroscience 'Rita Levi Montalcini', University of Turin.

\section{References and recommended reading}

Papers of particular interest, published within the period of review, have been highlighted as:

- of special interest

$\bullet$ of outstanding interest

1. Stanaway JD, Afshin A, Gakidou E, Lim SS, Abate D, Abate KH, Abbafati C, Abbasi N, Abbastabar H, Abd-Allah F et al.: Global, regional, and national comparative risk assessment of 84 behavioural, environmental and occupational, and metabolic risks or clusters of risks for 195 countries and territories, 1990-2017: a systematic analysis for the Global Burden of Disease Study 2017. Lancet 2018, 392:1923-1994.

2. Becker S, Fenton MJ, Soukup JM: Involvement of microbial components and toll-like receptors 2 and 4 in cytokine responses to air pollution particles. Am J Respir Cell Mol Biol 2002, 27:611-618.

3. Nemmar A, Hoet PHM, Vanquickenborne B, Dinsdale D, Thomeer M, Hoylaerts MF, Vanbilloen H, Mortelmans L, Nemery B Passage of inhaled particles into the blood circulation in humans. Circulation 2002, 105:411-414.

4. Li D, Li Y, Li G, Zhang Y, Li J, Chen H: Fluorescent reconstitution - $\quad$ on deposition of PM 2.5 in lung and extrapulmonary organs. Proc Natl Acad Sci U S A 2019, 116:2488-2493

Using a novel fluorescence imaging method, the authors show the distribution of (even single particles) PM2.5 in extrapulmonary organs with a high temporal and spatial resolution.

5. Oberdörster G, Sharp Z, Atudorei V, Elder A, Gelein R, Kreyling W, Cox C: Translocation of inhaled ultrafine particles to the brain. Inhal Toxicol 2004, 16:437-445.

6. Maher BA, Ahmed IAM, Karloukovski V, MacLaren DA, Foulds PG, Allsop D, Mann DMA, Torres-Jardón R, Calderon-Garciduenas L: Magnetite pollution nanoparticles in the human brain. Proc Natl Acad Sci U S A 2016, 113:10797-10801.

7. Campagnolo L, Massimiani M, Vecchione L, Piccirilli D, Toschi N, - Magrini A, Bonanno E, Scimeca M, Castagnozzi L, Buonanno G et al.: Silver nanoparticles inhaled during pregnancy reach and affect the placenta and the foetus. Nanotoxicology 2017 , 11:687-698

By means of transmission electron microscopy coupled with energydispersive X-ray spectroscopy and single-particle inductively coupled plasma mass spectrometry, the authors analyze the distribution of inhaled silver nanoparticles in maternal tissues and in the foetus. This is one of the few direct demonstrations that, in animal models, inhaled nanoparticles can reach the placenta and the foetus. 
8. Woodward N, Finch CE, Morgan TE: Traffic-related air pollution and brain development. AIMS Environ Sci 2015, 2:353-373.

9. Buoli M, Grassi S, Caldiroli A, Carnevali GS, Mucci F, lodice S, Cantone L, Pergoli L, Bollati V: Is there a link between air pollution and mental disorders? Environ Int 2018, 118:154-168.

10. Zhang $X$, Chen $X$, Zhang $X$ : The impact of exposure to air

- $\quad$ pollution on cognitive performance. Proc Natl Acad Sci U S A 2018, 115:9193-9197

By matching a longitudinal survey and air quality data in China, the authors examine the effect of both cumulative and acute exposures to air pollution for the same individuals over time on cognitive performance. They provide a well-documented demonstration that chronic exposure to air pollution impedes cognitive performance in verbal and math tests, and identify most vulnerable population cohorts.

11. Shehab MA, Pope FD: Effects of short-term exposure to particulate matter air pollution on cognitive performance. $\mathrm{Sci}$ Rep 2019, 9:1-10.

12. Zheng $X$, Wang $X$, Wang $T$, Zhang $H$, Wu H, Zhang $C$, Yu L, Guan $Y$ : Gestational exposure to particulate matter 2.5 (PM 2.5) leads to spatial memory dysfunction and neurodevelopmental impairment in hippocampus of mice offspring. Front Neurosci 2019, 13:1-18

13. Klocke C, Allen JL, Sobolewski M, Mayer-Pröschel M, Blum JL, Lauterstein D, Zelikoff JT, Cory-Slechta DA: Neuropathological consequences of gestational exposure to concentrated ambient fine and ultrafine particles in the mouse. Toxicol Sci 2017, 156:492-508.

14. Li K, Li L, Cui B, Gai Z, Li Q, Wang S, Yan J, Lin B, Tian L, Liu H et al.: Early postnatal exposure to airborne fine particulate matter induces autism-like phenotypes in male rats. Toxicol Sci 2018, 162:189-199.

15. Tseng CY, Yu JY, Chuang YC, Lin CY, Wu CH, Liao CW, Yang FH, Chao MW: The effect of Ganoderma microsporum immunomodulatory proteins on alleviating PM 2.5 -induced inflammatory responses in pregnant rats and fine particulate matter-induced neurological damage in the offsprings. Sci Rep 2019, 9:1-10.

16. Woodward NC, Haghani A, Johnson RG, Hsu TM, Saffari A,

- Sioutas C, Kanoski SE, Finch CE, Morgan TE: Prenatal and early life exposure to air pollution induced hippocampal vascular leakage and impaired neurogenesis in association with behavioral deficits. Transl Psychiatry 2018, 8:1-10

The authors show that, in rats, in utero and early life exposure to air pollution is associated with impaired contextual memory (novel object in context), reduced food-seeking behavior, and increased depressive behaviors (forced swim) at adult stages. This is accompanied by BBB breakdown and decreased numbers of newly generated neurons in the DG.

17. Fonken LK, Xu X, Weil ZM, Chen G, Sun Q, Rajagopalan S, Nelson RJ: Air pollution impairs cognition, provokes depressive-like behaviors and alters hippocampal cytokine expression and morphology. Mol Psychiatry 2011, 16:987-995

18. Wu G, Brown J, Zamora ML, Miller A, Satterfield MC, Meininger CJ, Steinhauser CB, Johnson GA, Burghardt RC, Bazer FW et al.: Adverse organogenesis and predisposed long-term metabolic syndrome from prenatal exposure to fine particulate matter. Proc Natl Acad Sci U S A 2019, 116:11590-11595.

19. Bolton JL, Marinero S, Hassanzadeh T, Natesan D, Le D,

- Belliveau C, Mason SN, Auten RL, Bilbo SD: Gestational exposure to air pollution alters cortical volume, microglial morphology, and microglia-neuron interactions in a sexspecific manner. Front Synaptic Neurosci 2017, 9:1-16

By analyzing mice prenatally exposed to DEP, the authors show an initia overexpansion of the cortex at late embryonic ages, which switched to decreased volume in young adult males. They also find increased microglial-neuronal interactions in DEP-exposed male offspring compared to other groups, suggesting that microglia activation mediates PM effects on brain growth.

20. Umezawa M, Onoda A, Korshunova I, Jensen ACØ, Koponen IK Jensen KA, Khodosevich K, Vogel U, Hougaard KS: Maternal inhalation of carbon black nanoparticles induces neurodevelopmental changes in mouse offspring. Part Fibre Toxicol 2018, 15:36.
21. Zhang $\mathrm{T}$, Zheng $\mathrm{X}$, Wang $\mathrm{X}$, Zhao $\mathrm{H}$, Wang $\mathrm{T}$, Zhang $\mathrm{H}$, Li W, Shen H, Yu L: Maternal exposure to PM 2.5 during pregnancy induces impaired development of cerebral cortex in mice offspring. Int J Mol Sci 2018, 19 pii: E257.

22. Klocke C, Allen JL, Sobolewski M, Blum JL, Zelikoff JT, Cory-

- Slechta DA: Exposure to fine and ultrafine particulate matter during gestation alters postnatal oligodendrocyte maturation, proliferation capacity, and myelination. Neurotoxicology 2018, 65:196-206

This paper shows that in utero exposure to fine and ultrafine PM in mouse results in persistent hypermyelination of the corpus callosum, due to the precocious engagement of oligodendroglia precursors into maturation, at the expenses of the amplification phase.

23. Allen JL, Oberdorster G, Morris-Schaffer K, Wong C, Klocke C, Sobolewski M, Conrad K, Mayer-Proschel M, Cory-Slechta DA: Developmental neurotoxicity of inhaled ambient ultrafine particle air pollution: parallels with neuropathological and behavioral features of autism and other neurodevelopmental disorders. Neurotoxicology 2017, 59:140-154.

24. Morris-Schaffer K, Merrill AK, Wong C, Jew K, Sobolewski M, Cory-Slechta DA: Limited developmental neurotoxicity from neonatal inhalation exposure to diesel exhaust particles in C57BL/6 mice. Part Fibre Toxicol 2019, 16:1-14.

25. Obernier K, Alvarez-Buylla A: Neural stem cells: origin, heterogeneity and regulation in the adult mammalian brain. Development 2019, 146 pii: dev156059.

26. Moreno-Jiménez EP, Flor-García M, Terreros-Roncal J, Rábano A,

-• Cafini F, Pallas-Bazarra N, Ávila J, Llorens-Martín M: Adult hippocampal neurogenesis is abundant in neurologically healthy subjects and drops sharply in patients with Alzheimer's disease. Nat Med 2019, 25:554-560

By analyzing human brain samples obtained under tightly controlled conditions, the authors show an abundant population of neuroblasts in the DG of neurologically healthy human subjects up to the ninth decade of life. This is one of the most recent papers describing the presence of immature neurons in the adult hippocampus, although the authors do not demonstrate that these neurons are generated during the adult life.

27. Sorrells SF, Paredes MF, Cebrian-Silla A, Sandoval K, Qi D,

-• Kelley KW, James D, Mayer S, Chang J, Auguste Kl et al.: Human hippocampal neurogenesis drops sharply in children to undetectable levels in adults. Nature 2018, 555:377-381

By analyzing the DG of human subjects at different ages, the authors show that the number of proliferating progenitors and young neurons declines sharply during the first year of life and only a few isolated young neurons persist by 7 and 13 years of age. In adult healthy adults, young neurons cannot be detected in the DG. Similar findings are obtained in the monkey hippocampus. Results of this paper support the view that adult hippocampal neurogenesis does not occur in humans.

28. Boldrini M, Fulmore CA, Tartt AN, Simeon LR, Pavlova I,

- Poposka V, Rosoklija GB, Stankov A, Arango V, Dwork AJ et al.: Human hippocampal neurogenesis persists throughout aging. Cell Stem Cell 2018, 22:589-599

By analyzing whole autopsy hippocampi from healthy (i.e. without cognitive impairment, neuropsychiatric disease, or treatment) human individuals ranging from 14 to 79 years of age, the authors find an abundant and comparable population of intermediate progenitors and of immature neurons in the DG of young and old subjects, with equivalent DG volume across ages. Yet, older individuals have a smaller quiescent progenitor pool. This is one of the most recent papers describing the presence of immature neurons in the adult hippocampus, although the authors do not demonstrate that these neurons are generated during the adult life.

29. Anacker C, Hen R: Adult hippocampal neurogenesis and cognitive flexibility-linking memory and mood. Nat Rev Neurosci 2017, 18:335-346.

30. Coburn JL, Cole TB, Dao KT, Costa LG: Acute exposure to diesel

- exhaust impairs adult neurogenesis in mice: prominence in males and protective effect of pioglitazone. Arch Toxicol 2018, 92:1815-1829

This paper provides a characterization of DEP effects on adult neurogenesis in the mouse SVZ/OB system and DG. The authors implicate microglia activation in these effects, since pioglitazone protected against DEP-induced alterations of hippocampal neurogenesis.

31. Brunekreef B, Harrison RM, Künzli N, Querol X, Sutton MA, Heederik DJ, Sigsgaard T: Reducing the health effect of particles from agriculture. Lancet Respir Med 2015, 3:831-832. 
32. Cheng L, Lau WKW, Fung TKH, Lau BWM, Chau BKH, Liang Y, Wang Z, So KF, Wang T, Chan CCH et al.: PM2.5 exposure suppresses dendritic maturation in subgranular zone in aged rats. Neurotox Res 2017, 32:50-57.

33. Bisht K, Sharma KP, Lecours C, Gabriela Sánchez M, El Hajj H, Milior G, Olmos-Alonso A, Gómez-Nicola D, Luheshi G, Vallières L et al:: Dark microglia: a new phenotype predominantly associated with pathological states. Glia 2016, 64:826-839.

34. Rolando C, Boda E, Buffo A: Immune system modulation of parenchymal and germinal neural progenitor cells in physiological and pathological conditions. In Neural Stem Cells and Therapy. Edited by Tao n. InTech; 2012:9789533079585413-440.

35. Brown GC, Neher JJ: Microglial phagocytosis of live neurons. Nat Rev Neurosci 2014, 15:209-216.

36. Allen JL, Liu X, Weston D, Prince L, Oberdörster G, Finkelstein JN, Johnston CJ, Cory-Slechta DA: Developmental exposure to concentrated ambient ultrafine particulate matter air pollution in mice results in persistent and sex-dependent behavioral neurotoxicity and glial activation. Toxicol Sci 2014, 140:160178.

37. Cole TB, Coburn J, Dao K, Roqué P, Chang YC, Kalia V, Guilarte TR, Dziedzic J, Costa LG: Sex and genetic differences in the effects of acute diesel exhaust exposure on inflammation and oxidative stress in mouse brain. Toxicology 2016, 374:1-9.

38. Onoda A, Takeda K, Umezawa M: Dose-dependent induction of astrocyte activation and reactive astrogliosis in mouse brain following maternal exposure to carbon black nanoparticle. Part Fibre Toxicol 2017, 14:1-16.

39. Pavanello S, Bonzini M, Angelici L, Motta V, Pergoli L, Hoxha M, Cantone L, Pesatori AC, Apostoli P, Tripodi A et al.: Extracellular vesicle-driven information mediates the long-term effects of particulate matter exposure on coagulation and inflammation pathways. Toxicol Lett 2016, 259:143-150.

40. Pergoli L, Cantone L, Favero C, Angelici L, lodice S, Pinatel E, Hoxha M, Dioni L, Letizia M, Albetti B et al.: Extracellular vesiclepackaged miRNA release after short-term exposure to particulate matter is associated with increased coagulation. Part Fibre Toxicol 2017, 14:32.

41. Raposo G, Stoorvogel W: Extracellular vesicles: exosomes, microvesicles, and friends. J Cell Biol 2013, 200:373-383.

42. Yao H, Ma R, Yang L, Hu G, Chen X, Duan M, Kook Y, Niu F, Liao K, Fu M et al.: MiR-9 promotes microglial activation by targeting MCPIP1. Nat Commun 2014, 5:4386.
43. Kawahara $\mathrm{H}$, Imai $\mathrm{T}$, Okano $\mathrm{H}$ : MicroRNAs in neural stem cells and neurogenesis. Front Neurosci 2012, 6:30.

44. Barca-Mayo O, Richard Lu Q: Fine-tuning oligodendrocyte development by microRNAs. Front Neurosci 2012, 6:13.

45. Jobe EM, Zhao X: DNA methylation and adult neurogenesis. Brain Plast 2016, 3:5-26.

46. Chang YC, Daza R, Hevner R, Costa LG, Cole TB: Prenatal and

- early life diesel exhaust exposure disrupts cortical lamina organization: evidence for a reelin-related pathogenic pathway induced by interleukin-6. Brain Behav Immun 2019, 78:105-115

This paper shows that perinatal exposure to DEP results in alterations of neuronal distribution within the cortical lamina, accompanied by upregulation of interleukin-6 and DNMT1, and decreased levels of reelin in adult mice. Since several polymorphisms of reelin are associated with Autism Spectrum Disorser (ASD) and reelin levels are low in ASD patients, such alterations are interpreted as the molecular substrates of the neurodevelopmental and behavioral effects of early life PM exposure.

47. Nawrot TS, Saenen ND, Schenk J, Janssen BG, Motta V,

- Tarantini L, Cox B, Lefebvre W, Vanpoucke C, Maggioni C et al.: Placental circadian pathway methylation and in utero exposure to fine particle air pollution. Environ Int 2018, 114:231241

By combining the estimation of daily PM 2.5 exposure levels and the analysis of the methylation of $\mathrm{CpG}$ sites within the promoter regions of the Circadian pathway genes, the authors show that 3rd trimester PM 2.5 exposure is associated with placental Circadian pathway methylation.

48. Neven KY, Saenen ND, Tarantini L, Janssen BG, Lefebvre W,

- Vanpoucke C, Bollati V, Nawrot TS: Placental promoter methylation of DNA repair genes and prenatal exposure to particulate air pollution: an ENVIRONAGE cohort study. Lancet Planet Health 2018, 2:e174-e183

By combining the estimation of daily exposure to different air pollutant and the analysis of the methylation of the promoter of key DNA repair and tumor suppressor genes, the authors show that PM2.5 exposure during pregnancy is associated with increased overall placental mutation rate and alterations in DNA repair gene methylation pattern.

49. Mckinnon PJ: Maintaining genome stability in the nervous system. Nat Neurosci 2013, 16:1523-1529.

50. Bouchard-Cannon P, Mendoza-Viveros L, Yuen A, Kærn M, Cheng HYM: The circadian molecular clock regulates adult hippocampal neurogenesis by controlling the timing of cellcycle entry and exit. Cell Rep 2013, 5:961-973.

51. Noda M, Iwamoto I, Tabata H, Yamagata T, Ito H, Nagata K: Role of Per3, a circadian clock gene, in embryonic development of mouse cerebral cortex. Sci Rep 2019, 9:5874. 